\title{
Some exact results for plane incompressible flow
}

\author{
Terry BROCKETT * \\ Department of Naval Architecture and Marine Engineering. The University of Michigan, \\ Ann Arbor, MI 48109, USA
}

Received 27 September 1988

\begin{abstract}
Simple results are derived from an exact conformal transformation of the flow field about a lifting circular cylinder at an angle of attack in incompressible flow to that about a profile. The mapping function is one proposed by Moriya several decades ago which stretches the field at great distances from the foil and is here used to derive a two-parameter family of symmetrical shapes. The geometry and flow speed are easily evaluated at arbitrary points on the profile surface and hence errors can be readily defined for evaluation of numerical predictions. The strength of equivalent contour distributions of sources, vortices and dipoles is also given.
\end{abstract}

\section{Introduction}

Exact solutions for incompressible potential flow about specific foils provide both general trends and a basis for error analysis when numerical methods are employed. Numerical solutions for two-dimensional flow about profiles are generally adequate but some questions remain relative to accuracy, efficiency, choice of formulation and robustness. Even three-dimensional procedures are often checked for proof-of-concept using two-dimensional solutions. If exact solutions are simple, they encourage quantitative error analysis (such as the a posteriori error measures defined by the sum of the absolute or squared error at a number of points, or the maximum error found over the discretized solution domain).

A classic procedure for defining exact solutions for two-dimensional fields is the conformal transformation of the known flow about a simple body, such as a circular cylinder, to that about the desired shape. Ashley and Landahl (1965) catalog a variety of transformations that have produced useful results. One transformation not included in their survey is that proposed by Moriya $(1938,1941)$. This transformation stretches and rotates the field at great distances from the profile as distinct from others. This stretching also occurs in the profile geometry mapping and elongates the shape in the chordwise direction, similar to other mappings. When the number of coefficients is great, this feature is irrelevant, but with an approximate analysis or a reduced number of terms - the stretching of the far field provides added flexibility in that convenient orientation of the foil is found together with a fixcd chordlength. This is in contrast to other transformations, such as that of Joukowsky (1910), for which evaluation of complex expressions are required to define the exact shape and surface speed for fixed chordlength. The Moriya transformation is specified as

$$
z=x+\mathrm{i} y=\frac{C_{-1} \zeta}{a}+C_{0}+\sum_{n=1}^{\infty} C_{n} \frac{a^{n}}{\zeta^{n}},
$$

\footnotetext{
* Present address: Hydrodynamics Research Associates, Inc., Great Falls, VA 22066, USA.
} 
where $z=x+\mathrm{i} y$ is the complex coordinate of a point in the field for the flow about a profile; $\zeta$ is the complex coordinate of a point in the field for flow about the simple configuration; $a$ is a characteristic dimension of the simple configuration, and $C_{n}=A_{n}+\mathrm{i} B_{n}, n \geqslant-1$, are complex transformation constants that map the simple body and flow field to that about the profile. The region far from the profile $(|z| \rightarrow \infty)$ is stretched by $\left|C_{-1}\right|=\left(A_{-1}^{2}+B_{-1}^{2}\right)^{1 / 2}$ and rotated by $\alpha_{0}=\tan ^{-1}\left(B_{-1} / A_{-1}\right)$; hence the free-stream is different between the simple and profile planes.

In addition to lift, moment, surface speed and geometry for selected foils, the strength of contour distributions of doublet, source, and vortex elements is presented and may also be useful in evaluation of numerical methods.

\section{Examples of exact-transformation profiles}

If the simple flow in the $\zeta$ plane were taken as that about a circular cylinder (with circulation), then with the four $C_{n} a^{n}$ consisting of only real terms, $A_{n} a^{n},-1 \leqslant n \leqslant 2$, a mapping of the cylinder, $\zeta=a \mathrm{e}^{\mathrm{i} \varphi}$, to a symmetrical profile $(x(\varphi)=x(2 \pi-\varphi), y(\varphi)=-y(2 \pi$ $-\varphi)$ ) produces the parametric profile coordinates

$$
\begin{aligned}
& x(\varphi)=A_{0}+\left(A_{-1}+A_{1}\right) \cos \varphi+A_{2} \cos 2 \varphi, \\
& y(\varphi)=\left(A_{-1}-A_{1}\right) \sin \varphi-A_{2} \sin 2 \varphi .
\end{aligned}
$$

The chordwise extent of the foil is maximum at $\varphi=0$ and $\pi$. We take the ordinate to be specified (below) as a two-parameter family $(\epsilon, \delta)$ and require that the remaining two free transformation constants be such that the chord lies between $1(\varphi=0)$ and zero $(\varphi=\pi)$. These conditions are satisfied by the following mapping coefficients given as combinations of the two parameters $(\epsilon, \delta)$ for which there is stretching but no rotation $\left(B_{-1}=0\right)$ of the far field between $z$ and $\xi$ :

$$
A_{-1}=(1+2 \epsilon) / 4, \quad A_{0}=(1-2 \epsilon \delta) / 2, \quad A_{1}=(1-2 \epsilon) / 4, \quad A_{2}=\epsilon \delta,
$$

then the profile specification (non-dimensionalized by the chordlength $c$ ) is:

$$
x(\varphi)=(1+\cos \varphi) / 2+\epsilon \delta(\cos 2 \varphi-1), \quad y(\varphi)=\epsilon(\sin \varphi-\delta \sin 2 \varphi),
$$

for which $0 \leqslant x(\varphi) \leqslant 1$, with the trailing edge located at $\varphi=0$ and the lcading edge at $\varphi=\pi$. It is clear that if the Joukowsky transformation (or an extended version of it) were employed (with $A_{-1}$ a fixed constant, independent of the foil), then only three (of the four) conditions would be met (typically, the nose, $x(\pi)$, is allowed to extend slightly upstream of the origin) or an increased number of mapping coefficients is required to have the chordlength extend from 0 to 1 .

A significant simplification for relating the $z$ and $\zeta$ planes is that the equation for $x(\varphi)$ can be inverted to find $\varphi(x)$ :

$$
\varphi(x)=\cos ^{-1} \frac{[1+16 \delta \epsilon(2 x+4 \epsilon \delta-1)]^{1 / 2}-1}{8 \delta \epsilon} .
$$

For $\delta=0, \epsilon=\frac{1}{2} \tau$ ( $\tau=$ thickness/chord ratio) the ellipse is defined in eq. (4) and for $\delta=\frac{1}{2}$, $\epsilon=2 \tau /(3 \sqrt{3})$, a shape similar to the symmetrical Joukowsky profile, with a cusped trailing edge, and maximum thickness at $\varphi=2 \pi / 3$, is obtained:

$$
x(\varphi)=(1+\cos \varphi) / 2+\frac{\tau}{3 \sqrt{3}}(\cos 2 \varphi-1), \quad y(\varphi)=\frac{2 \tau}{3 \sqrt{3}}\left(\sin \varphi-\frac{1}{2} \sin 2 \varphi\right) .
$$

For values of $\delta$ between 0 and $\frac{1}{2}$ a rounded trailing edge is specified and the shape is more like a conventional foil. An example of one of these intermediate foils is given by $(\epsilon=0.45127 \tau, \delta$ 


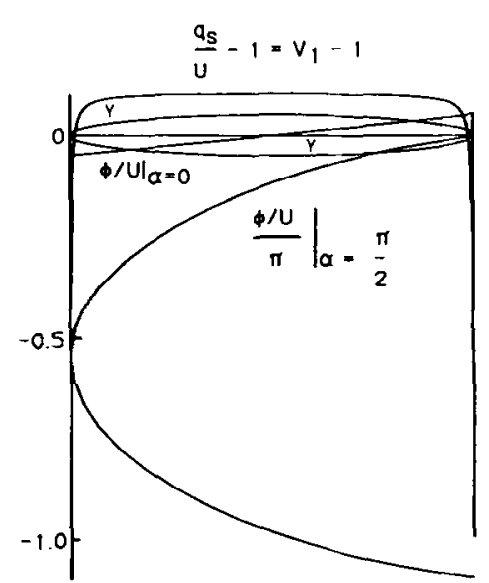

a

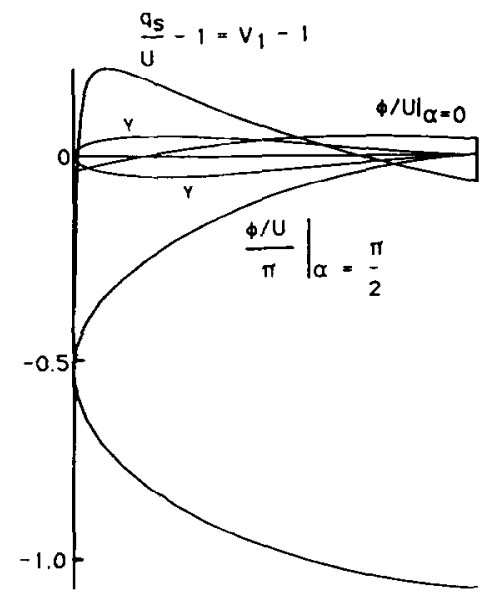

b

Fig. 1. Shape, surface speed and perturbation potential for (a) $10 \%$ ellipse and (b) $10 \%$ cusped-tail foils.

$=\frac{1}{4}$ ), which has a maximum of fset at $\varphi=1.94553$, or $x=0.29339$ and a rounded tail. Since the ellipse and cusped trailing-edge foil provide both a simple convex and a more complex inflected shape for error analysis with numerical methods, no further information is presented on such intermediate foils. The section shapes for the ellipse and cusped trailing-edge profile are shown in fig. 1 for $10 \%$ thick profiles.

The surface speed in the profile plane is obtained from an implicit differentiation of $F(\zeta)$, the complex potential for the flow about the circular cylinder with a stagnation point at $\varphi=0$ :

$$
q_{\mathrm{s}}=\left|\frac{\mathrm{d} F(\zeta)}{\mathrm{d} \zeta} \frac{1}{\mathrm{~d} z / \mathrm{d} \zeta}\right|_{\zeta=a \mathrm{e}} .
$$

Hence, the reference speed $U$ at great distances from the profile is:

$$
U=a V / A_{-1}
$$

where $V$ is the speed at great distances from the circular cylinder. The final expression for the non-dimensional surface speed (in the direction of increasing $\varphi$ ) is

$$
\frac{q_{s}}{U}=-\frac{\left(\frac{1}{2}+\epsilon\right)[\sin \varphi \cos \alpha+(1-\cos \varphi) \sin \alpha]}{\left[(\mathrm{d} x / \mathrm{d} \varphi)^{2}+(\mathrm{d} y / \mathrm{d} \varphi)^{2}\right]^{1 / 2}}=V_{1} \cos \alpha+V_{2} \sin \alpha .
$$

The fundamental solutions $V_{1}$ and $V_{2}$ (for $\alpha=0$ and $\alpha=\pi / 2$ respectively) can be linearly combined to give the surface speed at any angle of attack.

The lift coefficient is obtained from the circulation defined in $F(\zeta)$ :

$$
C_{\mathrm{L}}=\frac{\rho U \Gamma}{\frac{1}{2} \rho U^{2} c}=8 \pi A_{-1} \sin \alpha=2 \pi(1+2 \epsilon) \sin \alpha
$$

and the clockwise moment coefficient about an arbitrary point $\left(x_{0}, y_{0}\right)$ is:

$$
C_{\mathrm{m}}=\frac{M}{\frac{1}{2} \rho U^{2} c^{2}}=-4 \pi\left[\sin 2 \alpha A_{-1}\left(A_{0}-x_{0}-A_{1}\right)+(\cos 2 \alpha-1) A_{-1} y_{0}\right] .
$$

Hence the moment about the quarter chord $\left(\frac{1}{4}, 0\right)$ is:

$$
C_{\mathrm{m}}=-\pi \epsilon(1+2 \epsilon)(1-2 \delta) / 2 \sin 2 \alpha
$$


or the aerodynamic center (point on $x$ axis with fixed moment; equal to $C_{\mathrm{m}}=0$ for the present case) is

$$
x_{\mathrm{ac}}=A_{0}-A_{1}=\frac{1}{4}+\epsilon\left(\frac{1}{2}-\delta\right) .
$$

In some applications, the (real) scalar velocity potential $\Phi$ at points on the surface of the profile is of interest. The potential for the total flow field is:

$$
\Phi(\varphi) / U=\operatorname{Re}\left[\left.F(z(\zeta))\right|_{\zeta=a \mathrm{e}^{\mathrm{i} \varphi}}\right] / U=\left(\frac{1}{2}+\epsilon\right)[\cos (\varphi-\alpha)-\varphi \sin \alpha] .
$$

The (real) perturbation velocity potential $\phi$ at point on the profile surface is:

$$
\begin{aligned}
\phi / U & =\Phi(\varphi) / U-\cos \alpha x(\varphi)-\sin \alpha y(\varphi) \\
& =\left(\frac{1}{2}+\epsilon\right)[\cos (\varphi-\alpha)-\varphi \sin \alpha]-\cos \alpha x(\varphi)-\sin \alpha y(\phi),
\end{aligned}
$$

where the angular variable $\varphi$ is given by eq. (5) and $(x(\varphi), y(\varphi))$ points are specified in eq. (4), Plots of the perturbation potential at points on the body surface for $\alpha=0$ and $\pi / 2$ are also given in fig. 1.

\section{Singularity strength for contour distributions}

If a description of the flow field were sought to represent the perturbation potential in terms of the singularity strengths for insertion in Green's third identity, then the value of the perturbation potential at points on the body contour (the doublet strength) is given by eq. (15) and the strength of the source distribution $[\sigma=(\partial \phi / \partial n) / U]$ along the contour is:

$$
\sigma=\frac{1}{U} \frac{\partial \phi}{\partial n}=-\left(\cos \alpha n_{x}+\sin \alpha n_{y}\right)
$$

where $n_{x}$ and $n_{y}$ are components of the exterior unit normal $\boldsymbol{n}=\left(n_{x}, n_{y}\right)$. If $\boldsymbol{N}=\boldsymbol{T} \times \boldsymbol{k}$, where $\boldsymbol{T}=(\partial / \partial \varphi)\{x(\varphi) \boldsymbol{i}+y(\varphi) \boldsymbol{j}\}$ and $\boldsymbol{k}=\boldsymbol{i} \times \boldsymbol{j}$, then $\boldsymbol{n}=\boldsymbol{N} /|\boldsymbol{N}|$. A distribution of vortices on the contour - rather than dipoles - has a vector strength:

$$
\boldsymbol{\gamma}=\boldsymbol{\gamma} \boldsymbol{k}=\boldsymbol{n} \times \nabla \mu=\boldsymbol{n} \times\left(q_{\mathrm{s}} \boldsymbol{t}-U \boldsymbol{e}_{\alpha}\right) / U,
$$

where $q_{\mathrm{s}}$ is the surface speed given by eq. (9), $\nabla$ is the gradient operator, $\boldsymbol{t}=\boldsymbol{T} /|\boldsymbol{T}|$ is a unit vector tangent to the profile in the counterclockwise direction $(k=n \times t)$, and $e_{\alpha}=\cos \alpha \boldsymbol{i}+$ $\sin \alpha j$ is a unit vector along the direction of the far-field velocity vector. Lamb (1932) extends considcration of the ficld to points locatcd inside the contour (for which there is an arbitrary velocity potential $\left.\phi_{\mathrm{i}}\right)$ and defines both a modified doublet $(\mu)$ and a modified source $(\sigma)$ distribution along the body contour:

$$
\begin{array}{ll}
\mu=\left(\phi-\phi_{\mathrm{i}}\right)_{\mathrm{B}} / U & \text { for the doublet strength, } \\
\sigma=\boldsymbol{n} \cdot\left[\nabla\left(\phi-\phi_{\mathrm{i}}\right)\right]_{\mathrm{B}} / U=(1 / U) \partial\left(\phi-\phi_{\mathrm{i}}\right)_{\mathrm{B}} / \partial \mathrm{n} & \text { for the source strength, }
\end{array}
$$

where $\mathrm{B}$ denotes values on the contour. We treat the perturbation velocity potential and take the internal potential to be opposite that for the free stream:

$$
\phi_{\mathrm{i}}=-U(x \cos \alpha+y \sin \alpha) .
$$

This internal uniform stream brings the flow to rest inside the profile. The doublet and source strength along the contour are, respectively:

$$
\mu=\left(\frac{1}{2}+\epsilon\right)[\cos (\varphi-\alpha)-\varphi \sin \alpha]=\Phi / U, \quad \sigma=n \cdot\left[\left(q_{s} t-U \boldsymbol{e}_{\alpha}\right)+U \boldsymbol{e}_{\alpha}\right] / U=0 .
$$

Thus for this particular choice of $\phi_{\mathrm{i}}$ there is a distribution about the contour of only doublets 
with strength equal to the total potential on the surface. This doublet distribution may be converted to a vector vortex strength:

$$
\boldsymbol{\gamma}=\boldsymbol{n} \times(\nabla \mu)=q_{\mathrm{s}} \boldsymbol{k} .
$$

For the non-lifting case, the singularity distribution can be taken as only sources. To eliminate the presence of doublets or vortices, a potential for the internal field must be constructed to cancel the external perturbation potential on the body, which at zero angle of attack is:

$$
\phi_{\mathrm{B}}-\left[\left(\frac{1}{2}+\epsilon\right) \cos \varphi-x(\varphi)\right] U=\epsilon[\cos \varphi-\delta \cos 2 \varphi] U+C_{U} .
$$

For elliptic profiles (for which $\delta=0$ ), this is a straightforward construction. Let the internal potential, evaluated on the boundary for a general angle of attack, be:

$$
\begin{aligned}
\phi_{\mathrm{i}, \mathrm{B}} & =2 \epsilon U[x(\varphi) \cos \alpha+y(\varphi) \sin \alpha]_{\delta=0} \\
& =\tau U[(1+\cos \varphi) \cos \alpha+\tau \sin \varphi \sin \alpha] / 2,
\end{aligned}
$$

then to within a constant, the following strengths are found:

$$
\begin{aligned}
& \mu=\sin \alpha(1+\tau)[(1-\tau) \sin \varphi-\varphi] / 2, \\
& \sigma=-(1+\tau) n \cdot e_{\alpha}=-(1+\tau) \frac{\tau \cos \varphi \cos \alpha+\sin \varphi \sin \alpha}{\left(\sin ^{2} \varphi+\tau^{2} \cos ^{2} \varphi\right)^{1 / 2}} .
\end{aligned}
$$

Hence for $\alpha=0$, there is a distribution of only sources along the contour of the ellipse. Other non-lifting configurations may also be represented by a contour distribution of only sources, but the expression for the strength is lengthy.

For the circular cylinder, $\tau=1$, eq. (24) reduces to a linearly varying doublet strength (or constant vortex strength) as a function of arc length along the contour that results from the circulation about the circular cylinder:

$$
\mu=-\sin \alpha \varphi \quad \text { or } \quad \gamma=-2 \sin \alpha \boldsymbol{k}
$$

and a source distribution along the contour of strength:

$$
\sigma=-2 \cos (\varphi-\alpha)
$$

In several numerical procedures, a distribution of sources together with a special distribution of vortices is selected for the solution representation. A subdivision of the surface into $2 \mathrm{~N}$ panels on the contour may be selected with constant (or variable) singularity strengths across the panel. The discretized equations are often evaluated by specifying $2 \mathrm{~N}$ body boundary conditions and the Kutta condition that the body streamline leave the contour appropriately at the trailing edge. It is thus convenient to have $2 N+1$ unknowns. Giesing (1964) selects the source strength on each of the $2 \mathrm{~N}$ subdivisions of the contour and a constant vortex strength, $\gamma_{0}$, as the $2 N+1$ unknowns. Hence, the circular cylinder is the only configuration shape for which the present analysis provides an analytic comparison.

\section{Cambered foils}

Foil shapes with camber can also be easily derived for coefficients with $n \leqslant 2$ :

$$
\begin{aligned}
& x(\varphi)=A_{0}+\left(A_{-1}+A_{1}\right) \cos \varphi+A_{2} \cos 2 \varphi+\left(B_{1}-B_{-1}\right) \sin \varphi+B_{2} \sin 2 \varphi, \\
& y(\varphi)=B_{0}+\left(A_{-1}-A_{1}\right) \sin \varphi-A_{2} \sin 2 \varphi+\left(B_{1}+B_{-1}\right) \cos \varphi+B_{2} \cos 2 \varphi .
\end{aligned}
$$


If the four $A_{i}(-1 \leqslant i \leqslant 2)$ are the same as in eq. (3) and if the $B_{i}$ are chosen as follows:

$$
B_{-1}=-(f+\gamma) / 2, \quad B_{0}=f / 2+\gamma, \quad B_{1}=(f-\gamma) / 2, \quad B_{2}=-f / 2,
$$

then the profile specification (for $x(0)=1$ and $\mathrm{d} x / \mathrm{d} \varphi=0$ at $\varphi=0$ ) is:

$$
\begin{aligned}
& x(\varphi)=(1+\cos \varphi) / 2+\epsilon \delta(\cos 2 \varphi-1)+f\left(\sin \varphi-\frac{1}{2} \sin 2 \varphi\right), \\
& y(\varphi)=\epsilon(\sin \varphi-\delta \sin 2 \varphi)+f(1-\cos 2 \varphi) / 2+\gamma(1-\cos \varphi) .
\end{aligned}
$$

These equations have $x(\pi)=0$ but $\mathrm{d} x / \mathrm{d} \varphi=-2 f$ at $\varphi=\pi$. The minimum $x$ value occurs at $\varphi=\pi+\theta$ where $\theta \approx 4 f /(1+8 \epsilon \delta)$ and the parameter $\gamma$ can be selected to make $y=0$ there. Problems with chordlength not a unit value (i.e. $c=x(0)-x(\pi+\theta)$ ) and difficulty with defining $\varphi(x)$ reduce the accessibility of the results for cambered foils. We give only a few more details for this family. The surface speed is

$$
\frac{q_{\mathrm{s}}}{U}=-\frac{2\left|C_{-1}\right|\left[\sin \left(\alpha-\alpha_{0}-\varphi\right)-\sin \left(\alpha-\alpha_{0}\right)\right]}{\left[(\mathrm{d} x / \mathrm{d} \varphi)^{2}+(\mathrm{d} y / \mathrm{d} \varphi)^{2}\right]^{1 / 2}}
$$

and the lift coefficient is

$$
\begin{aligned}
C_{\mathrm{L}} & =8 \pi / c\left(A_{-1}^{2}+B_{-1}^{2}\right)^{1 / 2} \sin \left(\alpha-\alpha_{0}\right) \\
& =2 \pi / c\left[(1+2 \epsilon)^{2}+4(f+\gamma)^{2}\right]^{1 / 2} \sin \left(\alpha-\alpha_{0}\right),
\end{aligned}
$$

where

$$
\begin{aligned}
& \left|C_{-1}\right|=\left(A_{-1}^{2}+B_{-1}^{2}\right)^{1 / 2}=\left[(1+2 \epsilon)^{2}+(2 f)^{2}\right]^{1 / 2} / 4, \\
& \alpha_{0}=\tan ^{-1}\left(B_{-1} / A_{-1}\right)=\tan ^{-1}[-2(f+\gamma) /(1+2 \epsilon)] .
\end{aligned}
$$

Brockett (1965) presents some explicit calculations for selected foils but these shapes are generally not convenient because of the iteration required to produce data at selected chordwise stations.

\section{Summary}

Simple exact results for the flow about a class of symmetrical foils have been derived that are suitable for quantitative error analysis of numerical methods. The shape and flow variables at points on the foil contour are easily and directly obtained at arbitrary stations. The simplicity results from use of the Moriya transformation that stretches the solution domain far from the profile thus allowing an additional degree of freedom relative to more well known mappings when only a few terms are employed.

\section{References}

Ashley, H. and Landahl, M. (1965) Aerodynamics of Wings and Bodies (Addison-Wesley, Reading) pp. 51-57.

Brockett, T. (1965) Steady Two-Dimensional Pressure Distributions on Arbitrary Profiles, US Navy David Taylor Model Basin Report 1821 .

Giesing, J.P. (1964) Extension of the Douglas Neumann Program to Problems of Lifting Infinite Cascades, Douglas Aircraft Co., Report LB31653.

Joukowsky, N.E. (1910) Über die Konturen der Tragflächen der Drachenflieger, Z. Flugtech. Motorhuftsch. 1, 281.

Lamb, H. (1932) Hydrodynamics (Cambridge Univ. Press, Cambridge) pp. 59, 60.

Moriya, T. (1938) A Method of Calculating Aerodynamic Characteristic of an Arbitrary Wing Section, J. Soc. Aeron. Sri. Japan 5 (33), 7-17 (in Japanese).

Moriya, T. (1941) On the Aerodynamic Theory of an Arbitrary Wing Section, J. Soc. Aeron. Sci. Japan 8 (78), 1054-1060 (English translation in Selected Scientific and Technical Papers, University of Tokyo, 1959, pp. 48-59). 